\title{
Treatment of Infected Cardiac Implantable Electronic Devices
}

\author{
Abdulla Fakhro, MD ${ }^{1}$ Faryan Jalalabadi, MD ${ }^{1} \quad$ Rodger H. Brown, $\mathrm{MD}^{1} \quad$ Shayan A. Izaddoost, MD, PhD ${ }^{1}$ \\ ${ }^{1}$ Division of Plastic and Reconstructive Surgery, Baylor College of \\ Address for correspondence Shayan A. Izaddoost, MD, PhD, \\ Medicine, Houston, Texas \\ Semin Plast Surg 2016;30:60-65. \\ Department of Surgery, Baylor College of Medicine, 1977 Butler Blvd., \\ Suite E6.100, Houston, TX 77030 (e-mail: shayani@bcm.edu).
}

\author{
Abstract \\ Keywords \\ - cardiac implantable \\ electronic devices \\ - left ventricular \\ devices \\ - infection \\ - salvage therapy \\ - antibiotic beads
}

With their rising benefits, cardiac implantable electronic devices (CIEDs) such as pacemakers and left ventricular assist devices (LVADs) have witnessed a sharp rise in use over the past 50 years. As indications for use broaden, so too does their widespread employment with its attendant rise of CIED infections. Such large numbers of infections have inspired various algorithms mandating treatment. Early diagnosis of inciting organisms is crucial to tailoring appropriate antibiotic and or antifungal treatment. In addition, surgical debridement and explant of the device have been a longstanding modality of care. More novel therapies focus on salvage of the device by way of serial washouts and instilling drug-eluting antibiotic impregnated beads into the wound. The wound is then serially debrided until clean and closed. This technique is better suited to patients whose device cannot be removed, patients who are poor candidates for cardiac surgery, or patients who have failed conventional prior treatments.
Since their earliest application, cardiac implantable electronic devices (CIEDs), such as pacemakers, implantable defibrillators, and left ventricular assist devices (LVADs), have proven beneficial in the prevention of fatal cardiac-related disorders. ${ }^{1}$ The past 50 years have seen a sharp rise in cardiac implantable device use with a resultant decline in deaths from ischemic, myocardial, and cardiac rhythm causes. ${ }^{2}$ As the indications and guidelines governing CIED use broaden, so too does their widespread employment. ${ }^{3}$ The rate of CIED infections has mirrored their increased use with some studies suggesting that the rate of device infections has overshadowed their implantation rate. ${ }^{4}$ Infections pose a severe burden on patients, lead to significant health care costs and lengthy hospital stays, and may also lead to mortality. ${ }^{5}$ When compared with noninfectious cardiac device complications, pacemaker infections result in an 8.4- to 11.6-fold increase in mortality rates along with a mean hospitalization cost ranging from $\$ 31,149$ to $\$ 55,003$. ${ }^{6}$

Although both pacemakers and LVADs are implantable cardiac devices, their infection profile and treatment differ significantly due to the size of the device and the need for an external power source for the LVAD. However, both demonstrate a wide range of infection rates, with the true incidence of infection remaining elusive. ${ }^{7}$ Topkara et al report a pacemaker infection rate of between $13 \%$ and $80 \%$; however, others estimate it to be between $2 \%$ and $4 \%$ with rates rising $124 \%$ between years 1990 to 1999 and a 57\% rise from 2004 to 2006, respectively. ${ }^{8,9}$ Similarly, infection rates related to LVAD placement demonstrate a large range of between $13 \%$ and $80 \%$ among recipients. ${ }^{10}$

This wide variability in infection risk is in part due to different types of infections that have been included under the category of CIED-related infections. Various reports broadly included patients with surgical site infections, postoperative pneumonia, central venous catheter-related sepsis, and nosocomial urinary tract infections, in addition to infection of the CIED. ${ }^{10}$ Various comorbidities may contribute to CIED infections. Patients of advanced age, with congestive heart failure, with a metastatic malignancy, on corticosteroid therapy, or with renal failure are more likely to develop CIED infections thereby increasing their mortality. ${ }^{9}$

\section{Device Infection Diagnosis}

\section{Pacemakers}

The diagnosis of pacemaker infections is often challenging. Pocket site infections are diagnosed clinically, often presenting with inflammatory skin changes including pain, swelling,
Issue Theme Medical Device Infections; Guest Editor, Shayan A. Izaddoost, MD, PhD
Copyright @ 2016 by Thieme Medical Publishers, Inc., 333 Seventh Avenue, New York, NY 10001, USA. Tel: +1(212) 584-4662.
DOI http://dx.doi.org/ 10.1055/s-0036-1580733. ISSN 1535-2188. 


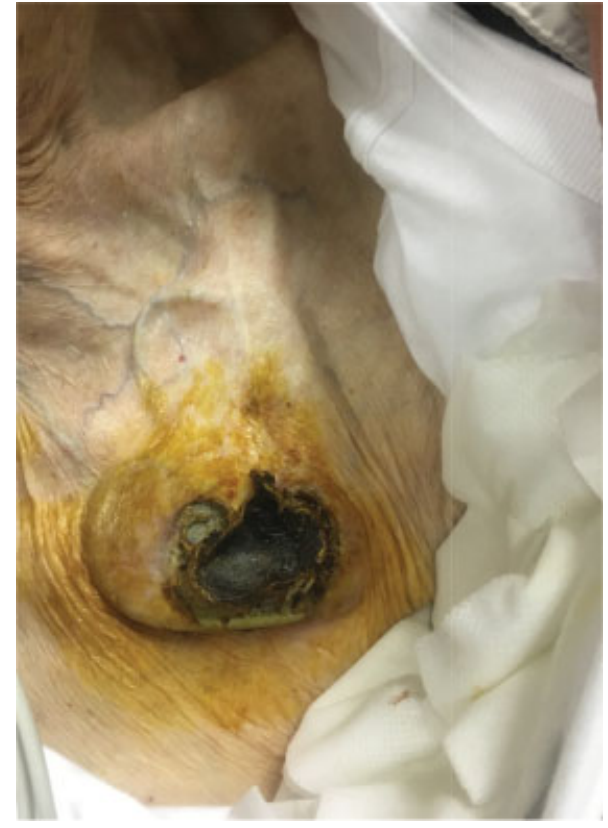

Fig. 1 An 82-year-old man with an exposed pacemaker with necrotic skin.

and redness. There may be skin and soft tissue ulceration and drainage. The first sign of infection may be erosion through the skin at the site of the implant pocket, with external exposure of the device with or without local inflammatory changes ( - Fig. 1) ${ }^{11}$ Fever and other signs of systemic toxicity are frequently absent; however, infective endocarditis may be present. A diagnosis of pacemaker infective endocarditis is based on clinical parameters, blood cultures, and echocardiographic findings. ${ }^{12}$ In cases of noninfective hematoma or seroma, device salvage may be undertaken (-Fig. 2).

One of the major challenges in diagnosis is the determination of the extent of the infection. On initial assessment, laboratory testing, blood, and exudate cultures as well as imaging modalities, including chest $\mathrm{X}$-rays, transthoracic and transesophageal echocardiography, and computed tomography (CT) scanning are used to identify whether the infection is limited to the device pocket or stems from an endocardial or peripheral source. ${ }^{13}$ However, in addition to such studies, clinical experience helps to guide treatment decisions.

Identification of the causative agent requires cultures of the pacemaker pocket site and blood cultures ( $\mathbf{- T a b l e ~} \mathbf{1}$ ). Tissue culture sensitivities are higher than swab cultures from the pocket site. However, up to $30 \%$ of patients with clinical signs of pacemaker infections have negative cultures. ${ }^{13}$ Additional gram staining, anaerobic and aerobic bacterial cultures, along with fungal cultures and staining should be sought as well as mycobacteria cultures, if the initial gram stain is negative. Usually, bacterial seeding occurs at the time of implantation, revision, or replacement of the device. Lastly, the pacemaker may become hematogenously infected in the case of a bacteremia due to another infection. ${ }^{10}$

\section{Left Ventricular Assist Devices}

Similar to pacemakers, determination of the extent of infection when faced with an LVAD infection is particularly difficult. Left ventricular assist device infections are classified into three categories: isolated driveline infections, pump pocket infections, and intravascular device infection or LVAD endocarditis. Patients present with an array of complaints including cellulitis, drainage from the LVAD driveline and possible exposure of the device. A CT scan is often employed to determine whether the infection is limited to the driveline or if it extends to the LVAD pocket, however, the true extent of infection can only be determined at the time of debridement. Fever, leukocytosis, and positive blood cultures can herald LVAD device endocarditis, which may ultimately respond only to device exchange.

\section{Microbiology of Device Infection}

\section{Pacemakers}

Cardiac device infections consist of a wide variety of organisms, with reports of polymicrobial infections between 7 to $15 \%$ of the time (see - Fig. 3). ${ }^{14-16}$ The most common organism found across multiple studies is the Staphylococcus species. Methicillin-resistant Staphylococcus aureus, methicillin-sensitive $S$. aureus, methicillin-resistant S. epidermidis, and methicillin-sensitive $S$. epidermidis contribute to more
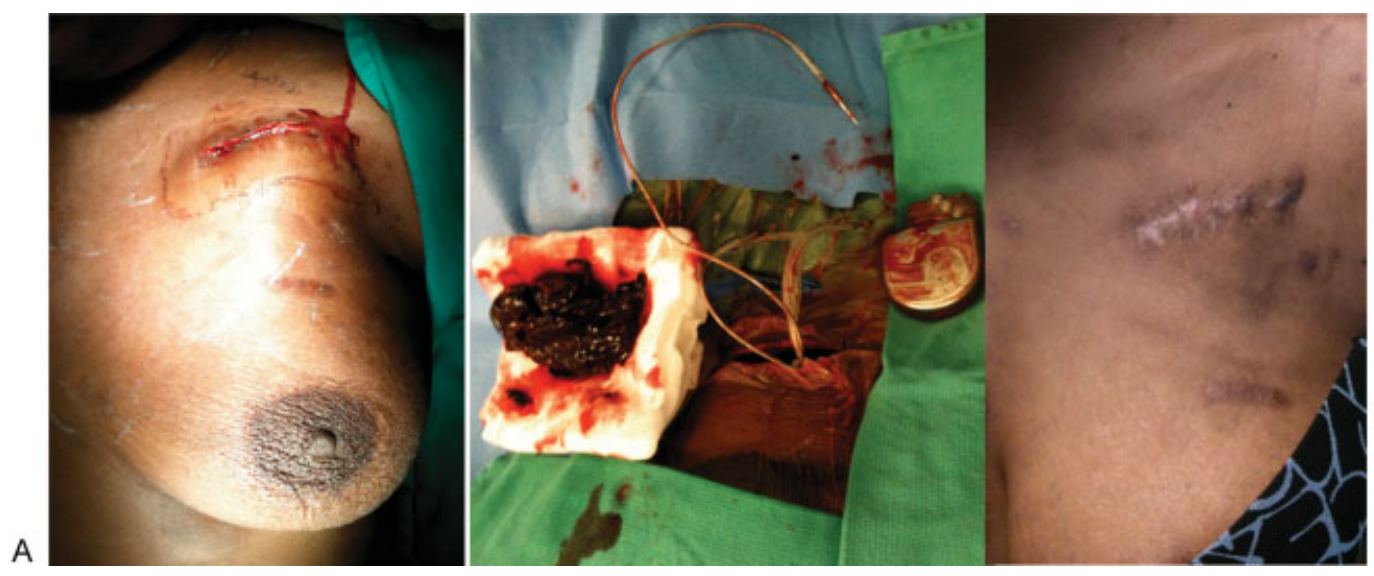

Fig. 2 (A) A 46-year-old woman with a hematoma. (B) Evacuation of hematoma and changing of generator. (C) Four months postoperative. 
Table 1 Causative agents behind pacemaker infections

\begin{tabular}{|l|l|}
\hline Microorganism & $\%$ Infections \\
\hline Coagulase-negative staph & 42 \\
\hline S. epidermidis & \\
\hline S. saprophyticus & \\
\hline S. schleiferi & \\
\hline S. lugdunensis & \\
\hline S. haemolyticus & \\
\hline Methicillin-sensitive S. aureus & 25 \\
\hline Gram-negative bacilli & 9 \\
\hline Enterobacteriaceae & \\
\hline Pseudomonas aeruginosa & \\
\hline Nonfermentative gram-negative bacilli & \\
\hline Polymicrobial & 7 \\
\hline Culture negative & 7 \\
\hline Methicillin-resistant S. aureus & 4 \\
\hline Gram-positive cocci & 4 \\
\hline Fungi & 2 \\
\hline
\end{tabular}

than half the pacemaker infection cases reported. Other gram-positives include Enterococcus faecalis, Enterobacter cloacae, Propionibacterium acnes, and Corynebacterium amycolatum; they make up $<5 \%$ of infections. Gram-negative organisms comprise approximately $10 \%$ of infections and include Pseudomonas aeruginosa, Klebsiella pneumoniae, Providencia stuartii, Serratia marcescens, Stenotrophomonas maltophilia, Enterobacter aerogenes, Escherichia coli, Citrobacter koseri, and others. Fungal agents, although rare, include Candida species and Aspergillus fumigatus (see - Fig. 3). ${ }^{14-16}$
Staphylococci and certain Candida infections are difficult to treat due to their ability to build biofilms on surfaces of foreign bodies such as CIEDs. Biofilms are a thick, multilayered film that mechanically traps bacteria, which when dormant, are highly resistant to bacteriocidal antibiotics via inhibition of cell wall biosynthesis, such as $\beta$-lactam antibiotics. $^{17-19}$

\section{Left Ventricular Assist Devices}

Similarly, LVAD infections may be attributed to various microorganisms. Driveline infections and pocket infections are mostly caused by gram-positive organisms, particularly the Staphylococcus and Enterococcus species. The most common gram-negative pathogen is Pseudomonas. Candida species may be attributed to driveline, pump-pocket, as well as LVAD-associated endocarditis in susceptible individuals. In addition, the majority of fungal pathogens may be drug resistant and be challenging to treat. $^{5}$

\section{Treatment of Device Infections}

Cardiac implantable electronic device explantation along with culture-driven intravenous (IV) antibiotics, remain the standard treatment modality in addressing device infections. ${ }^{14}$ Device salvage, as discussed below, is reserved for patients that are LVAD dependent and unable to tolerate explantation.

\section{Pacemakers}

The treatment of pacemaker infection consists of complete removal of the infected hardware and a capsulectomy followed by individualized antimicrobial therapy. ${ }^{15,20-25}$ If patients are pacemaker dependent, a temporary pacer is placed at or before the time of exchange. ${ }^{15,23}$ Reimplantation of devices, if necessary, depends on the location of the infection. Preferably, the pacemaker should be placed on

\section{Microbiology of Pacemaker Infections}

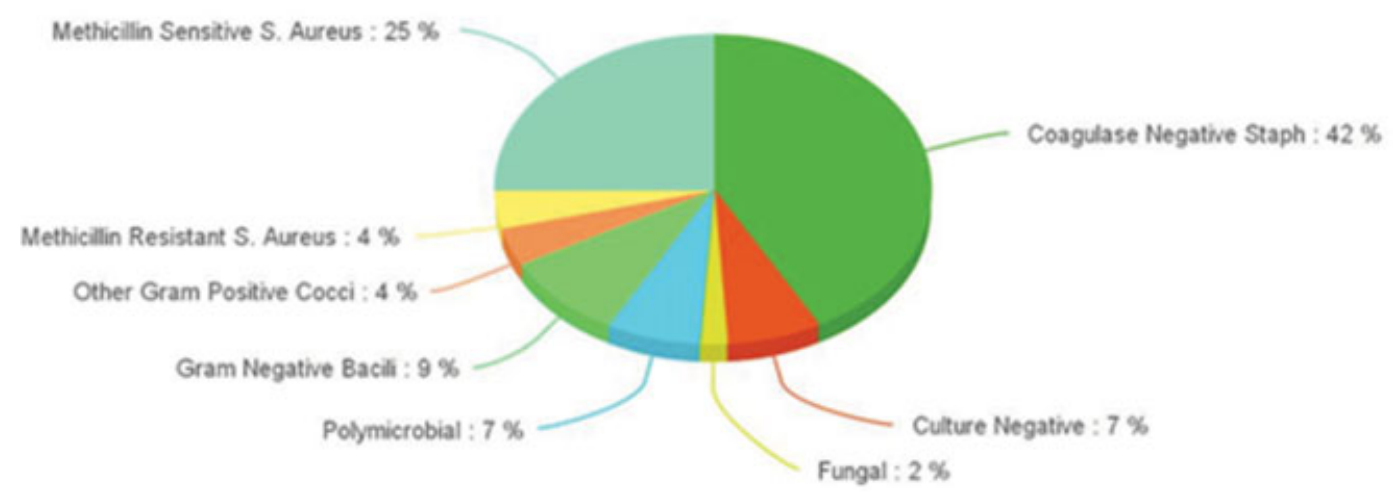

Coagulase Negative Staph Culture Negative $\quad$ Fungal Polymicrobial Gram Negative Bacili Other Cram Positive Cocci Methicillin Resistant S. Aureus Methicillin Sensitive S. Aureus

Fig. 3 The microbiology of pacemaker infections. 


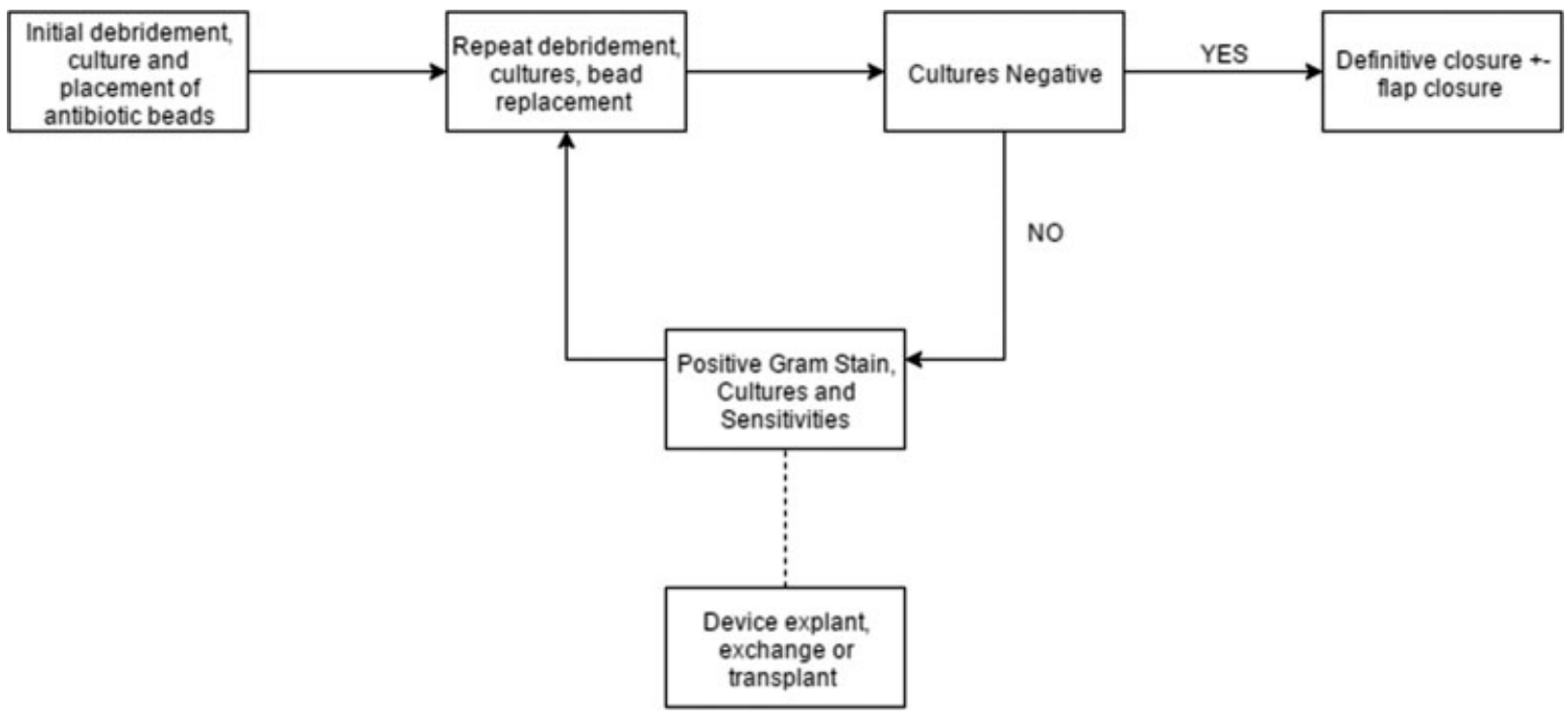

Fig. 4 Algorithm used for treatment of all patients. Repeated débridement and bead exchange were typically performed every 1 to 2 weeks until results of surgical-site cultures were negative or until other definitive endpoints were reached (i.e., device removal, transplantation, or death). ${ }^{5}$

A
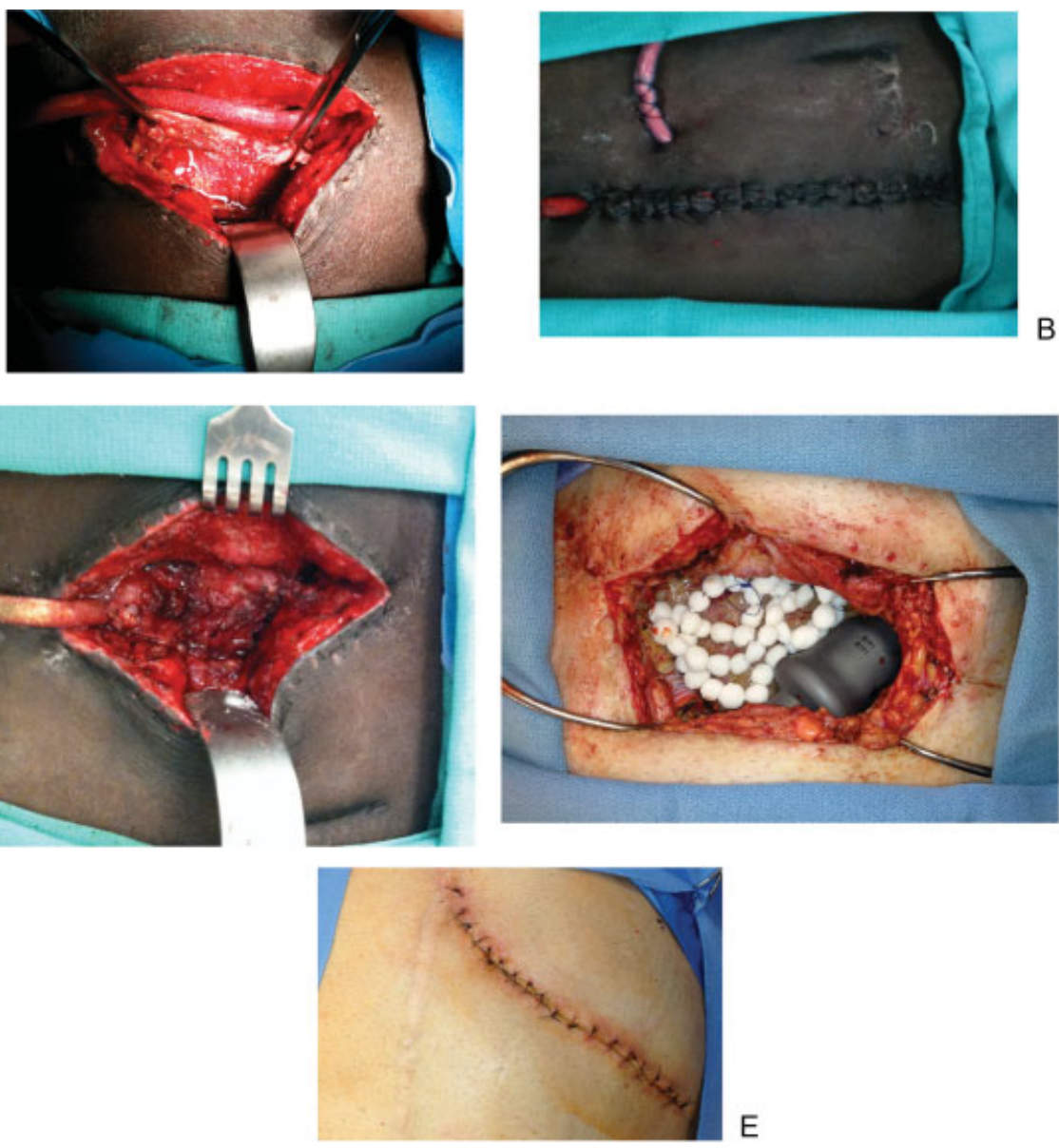

Fig. 5 (A-C) Patient undergoing coverage of salvaged left ventricular assist devices (LVAD) drive line using the anterior rectus sheath. (D, E) Placement of antibiotic beads for salvage of LVAD.

the contralateral side to the infection, after cellulitis has resolved and cultures are negative. Another strategy involves the placement of the device in a novel plane, often subpectoral. This ensures the device is covered by healthy vascularized muscle. It is particularly useful in emaciated or thin patients with poor overlying tissues and thin skin. This process is usually undertaken in a mean of 7 days. When infective endocarditis is present, timing is guided by negative blood cultures, a clinical assessment of the patient, and improved vegetation burden. ${ }^{15}$ 
Time to reimplantation using the traditional approach averages between 7 and 15 days, depending on the location of the infection and the presence/absence of cardiac devicerelated infective endocarditis (CDIE). Patients with CDIE have the longest time to reimplantation. The reported mortality rates in this population ranges from $13 \%$ to $21 \%{ }^{14,15,26}$ Complications that may arise when following the traditional method of device explantation include cardiac arrest, sepsis, operative cardiac tear, pulmonary embolism, hematoma of the device pocket site, pericardial effusions, pericarditis, venous or arterial thrombosis, and pneumothorax. Rodriguez et al reported fatal complications from extraction procedures in 2 of 506 patients. Tarakji et al reported fatal complications from device extraction in 2 of 412 patients, and a 1-year mortality rate of $17 \%$; $2.6 \%$ of patients who underwent reimplantation had relapsing infection. ${ }^{16}$

\section{Left Ventricular Assist Device Salvage}

Patients presenting with LVAD infections with recovering cardiac tissue are best treated with removal of the device. This can occur in the setting of cardiac recovery or in cases where a transplant heart becomes available. However, these procedures are not without inherent morbidity and mortality. Various techniques have been described to salvage infected LVADS, including IV antibiotics, wet to dry dressing changes around the device, and negative pressure wound therapy. Recently, a novel approach for device salvage of infected LVADs was developed for high-risk patients where the LVAD could not be removed, patients who were poor candidates for major cardiac surgery, or patients who had failed previous treatments. Salvage procedures involve surgical incision, drainage and aggressive debridement, and placement of antibiotic impregnated beads or slurry (-Fig. 4).

Using the algorithm above, Kretlow et al successfully cleared infections in 17 of 26 patients with left ventricular assist devices. Cleared infections led to a dramatic decline in mortality rates. For patients whose infection persisted, mortality rates were $67 \%$ ( 6 of 9 patients) over the course of the study, whereas that of the cleared population was $29 \%$ ( 5 of 17 patients). The cause of mortality in all those with persisting infections was sepsis, whereas the cause in those with cleared infections included cerebrovascular accident, right heart failure, left ventricular assist device-related gastrointestinal perforation, and graft rejection. ${ }^{5}$

Antibiotic-impregnated beads have been successfully used in the treatment of prosthetic-related infections by orthopedic and vascular surgeons with reported clearance rates ranging from $60 \%$ to $100 \%$ with recurrence rates ranging from 0 to $20 \% .^{22,27-31}$ The beads are capable of delivering high concentrations of necessary antibiotics directly to a site of infection, thereby reducing the systemic side effects. Beads are available in resorbable and nonresorbable materials. Nonresorbable beads require bead exchange or removal, unlike their resorbable counterparts.

Polymethylmethacrylate is a nonresorbable medium that is used across many subspecialties to deliver high concentrations of antibiotics locally. Its high cure temperature of $93^{\circ} \mathrm{C}$ requires the use of heat-stable antibiotics such as vancomycin, tobramycin, and gentamicin, which sometimes limits its use. ${ }^{31}$ However, if resistance to these antimicrobials is encountered, fibrin sealant or calcium sulfate impregnated with the susceptible heat-labile antibiotic of choice may be used. Kretlow et al encountered difficulty with this alternative regimen. Two of three patients treated with fibrin sealant or calcium sulfate-based beads had difficulty clearing infections. This was possibly due to the decreased efficacy of the fibrin sealant and calcium sulfate compared with polymethylmethacrylate; however, this may also have been due to the challenge of treating multidrugresistant organisms. $^{5}$

Repeated debridement and bead exchange typically performed every 1 to 2 weeks until surgical site cultures were negative or until other definitive endpoints had been reached (device removal, transplant, or if the patient expired). Subsequently, the device was permanently covered with a vascularized flap using a myocutaneous, fasciocutaneous, and/or omental flap.

The location of the infection ultimately played a role in treatment. Once cultures were negative, patients with infection at the driveline underwent repositioning of the driveline underneath the anterior rectus sheath ( - Fig. 5A-C). Infection of the device pocket ( - Fig. 5D, E) required local tissue coverage and rectus abdominis muscle flaps where soft tissue coverage was warranted. ${ }^{5}$

\section{Conclusion}

Although tremendous gains have been made in our understanding of the pathogenesis, risk factors, and management of CIED infections over the last decade, the burden on patients and the health care system represents a significant challenge. For these patients, early diagnosis can make a great difference in terms of survival. Intravenous antibiotics and nonsurgical approaches may not provide definitive treatment in some of these conditions, with many recommending extraction and device removal, although that is not without its attendant risks. Patients who are LVAD dependent and are unable to undergo major cardiac surgery may benefit from antibiotic bead placement and device salvage with outcomes comparable with those of the currently recommended treatments above.

\section{References}

1 Myerburg RJ. Implantable cardioverter-defibrillators after myocardial infarction. N Engl J Med 2008;359(21):2245-2253

2 Antman EM. Cardiovascular Therapeutics: A Companion to Braunwald's Heart Disease. 2nd ed. Philadelphia: WB Saunders; 2002

3 Goldberger Z, Lampert R. Implantable cardioverter-defibrillators: expanding indications and technologies. JAMA 2006;295(7): 809-818

4 Voigt A, Shalaby A, Saba S. Continued rise in rates of cardiovascular implantable electronic device infections in the United States: temporal trends and causative insights. Pacing Clin Electrophysiol 2010;33(4):414-419

5 Kretlow JD, Brown RH, Wolfswinkel EM, et al. Salvage of infected left ventricular assist device with antibiotic beads. Plast Reconstr Surg 2014;133(1):28e-38e 
6 Sohail MR, Henrikson CA, Braid-Forbes MJ, Forbes KF, Lerner DJ. Mortality and cost associated with cardiovascular implantable electronic device infections. Arch Intern Med 2011;171(20):1821-1828

7 Eggimann P, Waldvogel FA. Infections Associated with Indwelling Medical Devices: Pacemaker and Defibrillator Infections. 3rd ed. Washington, DC: ASM Press; 2000

8 Topkara VK, Kondareddy S, Malik F, et al. Infectious complications in patients with left ventricular assist device: etiology and outcomes in the continuous-flow era. Ann Thorac Surg 2010;90(4):1270-1277

9 Sastry S, Rahman R, Yassin MH. Cardiac implantable electronic device infection: from an infection prevention perspective. Adv Prev Med 2015;2015:357087

10 Baddour LM, Bettmann MA, Bolger AF, et al; AHA. Nonvalvular cardiovascular device-related infections. Circulation 2003; 108(16):2015-2031

11 Baddour LM, Cha YM, Wilson WR. Clinical practice. Infections of cardiovascular implantable electronic devices. N Engl J Med 2012; 367(9):842-849

12 Baddour LM, Epstein AE, Erickson CC, et al; American Heart Association Rheumatic Fever, Endocarditis, and Kawasaki Disease Committee; Council on Cardiovascular Disease in Young; Council on Cardiovascular Surgery and Anesthesia; Council on Cardiovascular Nursing; Council on Clinical Cardiology; Interdisciplinary Council on Quality of Care; American Heart Association. Update on cardiovascular implantable electronic device infections and their management: a scientific statement from the American Heart Association. Circulation 2010;121(3):458-477

13 Dy Chua J, Abdul-Karim A, Mawhorter S, et al. The role of swab and tissue culture in the diagnosis of implantable cardiac device infection. Pacing Clin Electrophysiol 2005;28(12):1276-1281

14 Sohail MR, Uslan DZ, Khan AH, et al. Management and outcome of permanent pacemaker and implantable cardioverter-defibrillator infections. J Am Coll Cardiol 2007;49(18):1851-1859

15 Rodriguez Y, Garisto J, Carrillo RG. Management of cardiac devicerelated infections: a review of protocol-driven care. Int J Cardiol 2013;166(1):55-60

16 Tarakji KG, Chan EJ, Cantillon DJ, et al. Cardiac implantable electronic device infections: presentation, management, and patient outcomes. Heart Rhythm 2010;7(8):1043-1047

17 Nagpal A, Baddour LM, Sohail MR. Microbiology and pathogenesis of cardiovascular implantable electronic device infections. Circ Arrhythm Electrophysiol 2012;5(2):433-441

18 Passerini de Rossi B, Feldman L, Pineda MS, Vay C, Franco M. Comparative in vitro efficacies of ethanol-, EDTA- and levofloxacin-based catheter lock solutions on eradication of Stenotrophomonas maltophilia biofilms. J Med Microbiol 2012;61(Pt 9): 1248-1253

19 Meyer C, Bierbaum G, Heidrich C, et al. Nucleotide sequence of the lantibiotic Pep5 biosynthetic gene cluster and functional analysis of PepP and PepC. Evidence for a role of PepC in thioether formation. Eur J Biochem 1995;232(2):478-489

20 Monkowski DH, Axelrod P, Fekete T, Hollander T, Furukawa S, Samuel R. Infections associated with ventricular assist devices: epidemiology and effect on prognosis after transplantation. Transpl Infect Dis 2007;9(2):114-120

21 Toda K, Yonemoto Y, Fujita T, et al. Risk analysis of bloodstream infection during long-term left ventricular assist device support. Ann Thorac Surg 2012;94(5):1387-1393

22 Benaerts PJ, Ridler BM, Vercaeren P, Thompson JF, Campbell WB. Gentamicin beads in vascular surgery: long-term results of implantation. Cardiovasc Surg 1999;7(4):447-450

23 Deharo JC, Quatre A, Mancini J, et al. Long-term outcomes following infection of cardiac implantable electronic devices: a prospective matched cohort study. Heart 2012;98(9):724-731

24 Franceschi F, Thuny F, Giorgi R, et al. Incidence, risk factors, and outcome of traumatic tricuspid regurgitation after percutaneous ventricular lead removal. J Am Coll Cardiol 2009;53(23): 2168-2174

25 Nishimura RA, Carabello BA, Faxon DP, et al; American College of Cardiology/American Heart Association Task Force. ACC/AHA 2008 guideline update on valvular heart disease: focused update on infective endocarditis: a report of the American College of Cardiology/American Heart Association Task Force on Practice Guidelines: endorsed by the Society of Cardiovascular Anesthesiologists, Society for Cardiovascular Angiography and Interventions, and Society of Thoracic Surgeons. Circulation 2008;118(8):887-896

26 Wazni O, Epstein LM, Carrillo RG, et al. Lead extraction in the contemporary setting: the LExICon study: an observational retrospective study of consecutive laser lead extractions. J Am Coll Cardiol 2010;55(6):579-586

27 Stone PA, Mousa AY, Hass SM, et al. Antibiotic-loaded polymethylmethacrylate beads for the treatment of extracavitary vascular surgical site infections. J Vasc Surg 2012;55(6):1706-1711

28 Scott DM, Rotschafer JC, Behrens F. Use of vancomycin and tobramycin polymethylmethacrylate impregnated beads in the management of chronic osteomyelitis. Drug Intell Clin Pharm 1988;22(6):480-483

29 Stone PA, Back MR, Armstrong PA, et al. Evolving microbiology and treatment of extracavitary prosthetic graft infections. Vasc Endovascular Surg 2008;42(6):537-544

30 Tintle SM, Forsberg JA, Potter BK, Islinger RB, Andersen RC. Prosthesis retention, serial debridement, and antibiotic bead use for the treatment of infection following total joint arthroplasty. Orthopedics 2009;32(2):87

31 Shi M, Kretlow JD, Nguyen A, et al. Antibiotic-releasing porous polymethylmethacrylate constructs for osseous space maintenance and infection control. Biomaterials 2010;31(14):4146-4156 